Samuel Mejía Restrepo 1

Alba Lucía Vélez Arango 2

Olga Clemencia Buriticá Arbol eda 3

María Cristina Arango Mejía 3

Jaime Al berto del Río Gómez 1

\section{La política farmacéutica nacional en Colombia y la reforma de la seguridad social: acceso y uso racional de medicamentos}

\author{
National pharmaceutical policy and social \\ security reform in Colombia: access and rational \\ use of medicines
}

1 Hospital de Caldas E.S.E. Carrera 25 Calle 48, Manizales, Colombia samere9@hotmail.com 2 Programa Nacional de Investigación en Salud Pública y Sistemas de Salud, Departamento de Salud Pública, Universidad de Caldas. Carrera 25 № 48 57, Apartado Aéreo 275, Manizales, Colombia. albalu@col 12.telecom.com.co 3 Departamento de Ciencias Básicas de la Salud, Universidad de Caldas. Calle 65 № 26 - 10, Manizales, Colombia. olclebuar@netcape.net
Abstract Based on the new social security system in Colombia (1993), which establishes equity and mandatory care as the basis for public heal th care provision, the authors analyze whether the formulation and implementation of pharmaceutical policy promote accessibility, availability, and rational use of medicines, thereby contributing to equity in health. Two approaches were used: a macro approach centered on the legal framework and various actors in the reform process and a micro approach related to the processes and results in the drug supply system. The authors studied the legal instruments backing the country's pharmaceutical policy and evaluated their application, using indicators and a specific di sease (diabetes mellitus) as a marker. Although there is a legal framework providing the people's right to access heal th care services and essential medicines, the country lacks a comprehensive pharmaceuticals policy. Most of the institutions experience problems in distributing the medicines listed under the Mandatory Health Plan, a low percentage of medicines is dispensed at zero cost, and a major portion of patients purchase medicines through associations of diabetics or rely on al ternative medicine. The study unveiled several obstacles to equity in health care coverage and access to essential medicines. Key words Equity; Social Security Reform in Health Care; Drug Utilization; Essential Drugs

Resumen A partir del nuevo sistema de seguridad social en Colombia (1993) que consagra la equidad y la obligatoriedad como fundamentos del servicio público, se analiza si la formulación y la implementación dela política farmacéutica favorecen la accesibilidad, la disponibilidad y el uso racional de los medicamentos, contribuyendo a la equidad en salud. Se utilizaron dos enfoques: uno macro, referido al análisis del marco jurídico y a los actores de la reforma, y otro mi cro, relaci onado a los procesos y resultados en el sistema de provisionamiento de medicamentos. Se estudiaron los instrumentos legal es que dan soporte a la política farmacéutica y fue evaluada su aplicación, utilizando indicadores y una enfermedad trazadora (diabetes mellitus). Aunque existe un marco jurídico que consagra el derecho al acceso de la población a servicios de salud y medicamentos esenciales, no hay una política de medicamentos integral. La mayoría delas instituciones presentan problemas para distribuir los medicamentos que pertenecen al Plan Obligatorio de Salud, un bajo porcentaje de medicamentos es obtenido sin costo y una gran parte de pacientes los adqui ere en asoci aci ones de diabéti cos o recurre a medicina alternativa. Se encontraron aspectos que desfavorecen la equidad en la cobertura en salud y la accesi bi lidad a medicamentos esenciales Palabras clave Equidad; Reforma dela Seguridad Social en Salud; Utilización de Medicamentos; Medicamentos Essenciales 


\section{Introducción}

Este artículo presenta un análisis de la Política Farmacéutica Nacional (PFN) en Colombia, situándola en el contexto de la reciente reforma e implantación del Sistema General de Seguridad Social en Salud (SGSSS). Colombia inicialmente ha formulado una PFN durante el período 1995-1998, como parte de la estrategia global de mejora de la atención de la salud que privilegia el enfoque de promoción y prevención. Esta política busca el logro de una oferta plural de medicamentos esenciales, con parámetros de calidad, oportunidad y precios asequibles, en el lugar y en el momento que el usuario los necesite, así como fomentar la educación al usuario de los servicios farmacéuticos y promover el uso racional de los medicamentos.

El nuevo SGSSS fue creado en 1993, mediante la Ley 100/ 93 (Colombia, 1993), la cual estableció también la reforma del sector de servicios de salud. Entre los principios que orientan la reforma, la equidad ocupa un lugar preeminente, y es entendida como la provisión gradual de servicios de salud de igual calidad a todos los habitantes de Colombia, independiente de su capacidad de pago (Colombia, 1993).

Con la reforma, se implementó un sistema cuya administración y financiamiento se articulan en dos modalidades: los regímenes contributivo y subsidiado. Al régimen contributivo accede el sector poblacional con capacidad de pago o cotizante inserto en el mercado formal de trabajo: Ios servidores públicos, pensionados y trabajadores independientes, con aportes proporcionales a sus ingresos. Este régimen es financiado mediante subsidios cruzados entre diferentes capacidades de pago y riesgo, es decir, entre quienes tienen mayores ingresos y menores riesgos, en favor de quienes tienen menores ingresos y mayores riesgos, buscando la equidad contributiva y distributiva. Al régimen subsidiado acceden los grupos poblacionales situados por debajo de una definida línea de pobreza, o sea el grupo de población de poca o ninguna accesibilidad económica, categorizada como población pobre y vulnerable. Los subsidios se entregan a través del Fondo de Solidaridad y Garantía, compuesto por $1 \%$ del valor de las cotizaciones del régimen contributivo y por otros aportes estatales y municipales de carácter público, y se otorgan por la vía de la demanda. La ley estipula, además, una categoría especial de "vinculados", integrada por personas que no están incluidas en ninguno de los dos regímenes anteriores, sea por motivos de incapacidad de pago o por otras razones $y$, mientras no logren ser beneficiarios formales del sistema, tendrán derecho a los servicios de atención en salud prestado por las instituciones públicas y privadas que tengan contrato con el Estado.

En síntesis, el nuevo sistema es financiado mediante mecanismos diferenciados de pagos, pretendiendo la solidaridad entre distintos grupos sociales, y subsidios, incluyendo también distintos copagos, cuotas moderadoras y cuotas de recuperación de costos, respetando la capacidad de pago de los afiliados (Colombia, 1993). La idea de la Ley 100 es que los participantes de los regímenes subsidiados y vinculados puedan acceder gradativamente al régimen contributivo.

Cada participante de los distintos regímenes debe tener una afiliación obligatoria a una Empresa Promotora de Salud (EPS), y el individuo cotizante y su familia se hacen acreedores a un plan de beneficios, el Plan Obligatorio de Salud (POS). Los "subsidiados" deben afiliarse a entidades específicas y se define un paquete básico de atención padronizado.

Una de las áreas de mayor sensibilidad para el cumplimiento de la Ley 100/ 93 es la relacionada a la política de medicamentos. En ese sentido, la ley contempla la provisión de medicamentos esenciales incluidos en el listado básico del POS (Colombia, 1993), y crea el Instituto Nacional de Vigilancia de Medicamentos y Alimentos - INVIMA (Colombia, 1993, 1994, 1995), buscando garantizar a la población beneficiaria de los regímenes contributivo y subsidiado el acceso equitativo a los medicamentos esenciales.

Sin embargo, esta política ha generado inquietudes, debido a que deja por fuera del mercado del SGSSS muchos productos que, a juicio de los profesionales o de los laboratorios productores, son de real utilidad para los beneficiarios; los usuarios deben adquirir los medicamentos no esenciales con dinero de su propio bolsillo; los medicamentos pueden ser suministrados, eventualmente, por las EPSs, con cargo a los planes complementarios. Esta situación plantea mayores inquietudes en el régimen subsidiado y en la población vinculada, afectando, sin duda, el principio de equidad.

En 1990, un estudio sectorial de salud (Yepes, 1990) describió las inequidades en el sector salud en Colombia. Primero, documentó la diferencia sistemática en el estado de salud de los individuos en relación con el ingreso. La mortalidad infantil entre los individuos de menor ingreso era tres veces mayor que la mortalidad infantil entre los individuos de mayor ingreso. De igual manera, la esperanza de vida 
era menor y la mortalidad general y la prevalencia de cualquier enfermedad eran consistentemente más altas entre los individuos de bajos ingresos. En segundo término, el estudio evidenció el menor uso de servicios de salud frente a una necesidad identificada entre las personas de menor ingreso. Oróstegui (1990) refiere un mayor índice de demanda rechazada por los prestadores de servicios entre las personas de bajos ingresos. Por otro lado, Molina (1993) encontró que, entre todos los servicios de salud prestados, las instituciones del Sistema Nacional de Salud cubrían tan sólo el $27 \%$ de la población, cuando el $47 \%$ estaba clasificada como pobre. Adicionalmente, el gasto en salud representaba el $1,5 \%$ de los ingresos de los individuos con mayores rentas y $7,5 \%$ entre los individuos con menores ingresos.

Otros estudios analizaron los hallazgos de esos autores (Tono, 2000): el 24\% de la población no recibía atención, ante una necesidad identificada, y el 55\% de las personas de bajos ingresos que no utilizó servicios frente a una necesidad no lo hacía por no poder cubrir los costos de los servicios. Menos del $50 \%$ de la población asalariada, y tan sólo el $23 \%$ de la población total, se encontraba afiliada a la seguridad social. Estas condiciones de acceso insuficiente e inequitativo, originadas por las bajas coberturas de aseguramiento y por los obstáculos económicos de la población, repercutían también en el acceso a los medicamentos y justificaron la necesidad de la reforma.

Con relación al área de política farmacéutica, Colombia ha tenido una historia importante. Fue uno de los primeros países en América Latina en implementar el registro sanitario (1927) y en contar, desde entonces, con una Comisión de Especialidades Farmacéuticas, hoy sala de medicamentos de la Comisión Revisora del INVIMA. En 1948, elaboró un listado de medicamentos prioritarios, cuya finalidad era garantizar la provisión de un grupo de medicamentos a toda la población. En 1975, elaboró el primer formulario nacional de medicamentos del país. En 1982, el Ministerio de Salud (MS) presentó los lineamientos de una política orientada al control de la calidad de los medicamentos y, en 1988, lanzó el Programa Nacional de Medicamentos Esenciales, con la asistencia técnica de la Organización Panamericana de la Salud (OPS) y la Organización Mundial de la Salud (OMS).

A pesar de estas iniciativas, gran parte de la población seguía sufriendo la falta de acceso a los medicamentos esenciales, los costos de adquisición eran con frecuencia innecesariamente altos y el conocimiento sobre una prescrip- ción racional y el uso apropiado de los medicamentos por prescriptores y consumidores continuaba inaceptablemente bajo.

Considerando estos problemas, en el nuevo SGSSS se incluyeron algunos lineamientos de PFN, entre ellos:

1) La elaboración del Listado Básico de Medicamentos, bajo responsabilidad del Consejo Nacional de Seguridad Social en Salud (CNSSS) (MS, 1997a).

2) La incorporación de la provisión de medicamentos esenciales bajo su denominación genérica en el POS, para los regimenes subsidiado y contributivo, garantizando la disponibilidad de alrededor de 300 medicamentos esenciales.

3) La creación de la Comisión Nacional de Precios de Medicamentos, con la función de definir la política en esta materia. Asignando al Ministerio de Desarrollo el seguimiento y el control de precios de los medicamentos.

4) La organización del Sistema Obligatorio de Garantía de Calidad del SGSSS (Colombia, 1996), para garantizar a los usuarios de los servicios el mayor beneficio, a un costo razonable y con el mínimo riesgo posible.

5) La creación de la Subdivisión de Servicios Farmacéuticos y del Laboratorio del MS, con el fin de fortalecer el desarrollo de la atención farmacéutica.

6) La creación del INVIMA, con el objetivo de ejecutar las políticas emanadas por el MS en materia de vigilancia sanitaria y de control de calidad de los medicamentos, alimentos, cosméticos, insecticidas de uso doméstico e insumos médicos.

7) La modificación de la legislación sobre registro sanitario y fabricación de productos farmacéuticos.

Sin embargo, se han detectado dificultades para la aplicación de la política de medicamentos. El MS (1995:3) afirmaba que "existe inequidad en la disponibilidad de medicamentos en grupos de población con baja capacidad adquisitiva o residentes en zonas rurales o de población dispersa", y esto se agrava considerando que la población menos favorecida generalmente sacrifica otras necesi dades básicas para adquirir medicamentos; según Instituto Nacional de Salud (INS, 1980), los más pobres, proporcionalmente, gastan mucho más en medicamentos.

En otro documento, el MS expresaba que "falta la formulación y universalización del concepto de atención farmacéutica y personal farmacéutico capacitado para una atención de cobertura nacional, [se observan] ... debilidades en la infraestructura disponible (planta física, equi pamiento), y en la formación y capaci- 
tación de los recursos humanos que prestan los servicios, así como se resalta el bajo desarrollo tecnológi co existente (indicadores de calidad, eficiencia, control y gestión), y la fal ta de protocolos de atención". Destacaba también la falta de información del consumidor y la exagerada disponibilidad de los medicamentos en un medio de libre comercialización, expresando que “es preocupantela generalizada creencia de la población, que toda enfermedad debe curarse con medicamentos y que, como suel e suceder con los bienes de consumo, el mayor bienestar necesariamente está relacionado con un mayor consumo de medicamentos". Como resultado de esta creencia, se tiende a "medicalizar la atención dela salud, relegando a un segundo plano la promoción y la prevención, así como la responsabilidad quetieneel mismo paciente yla comunidad en general para adoptar estilos de vida más saludables" (MS, 1996:6).

Partiendo de estas constataciones, este estudio buscó analizar la repercusión de la PFN sobre la equidad en el nuevo sistema de salud, a partir del impacto de su implementación sobre la accesibilidad y el uso racional de medicamentos. En esta perspectiva, se formularon Ios siguientes planteamientos: ¿El nuevo marco jurídico, así como los diferentes pronunciamientos gubernamentales que orientan el objetivo del sector salud, tiene como espíritu la reducción de las desigualdades? ¿Cómo se favorece dentro del sistema actual de prestación de servicios de salud el acceso a los mismos por la población más pobrey vulnerable? ¿Cómo se instrumentaliza la equidad en la accesibilidad y en el uso racional de los medicamentos?

Para responder a esas interrogantes se hizo un análisis en dos niveles: macro y micro. El análisis macro del marco jurídico mostró la existencia de normatividad que consagra el principio de equidad y el derecho universal a una atención en salud con calidad así como el acceso a los medicamentos esenciales, constatándose, por otro lado, la dispersión y la fragmentación de la regulación referida a la PFN. Los actores que participaron en la formulación de la PFN destacan aspectos que desfavorecen la aplicación de la política, enfatizando la ausencia de mecanismos de control por parte del Estado, entre otros. A nivel micro, el acceso a los medicamentos esenciales por los pacientes diabéticos se ve influenciado por el tipo de afiliación al sistema, observándose también dificultades para la obtención de los medicamentos por el paciente. Entretanto, la racionalidad en el uso de medicamentos presenta algunos avances. Finalmente, se presentan algunas recomendaciones.

\section{Metodología}

Esta investigación utilizó datos cuantitativos y cualitativos y fue desarrollada en dos niveles: un nivel macro, donde se analizó la política nacional; y un nivel micro, donde se analizaron los resultados de esa implementación en dos ciudades, consideradas polares.

Teniendo como eje de análisis la equidad en el acceso a los servicios de salud y a los medicamentos esenciales, se articulan los niveles macro y micro de la PFN implementada en CoIombia en el ámbito del SGSSS. En su dimensión estructural (o sea, la nueva forma de organización del sistema y de definiciones de los derechos de los ciudadanos), Ia PFN es analizada en relación a las bases legales que la sustentan y le dan viabilidad, considerando el contenido de la normatividad y la percepción de los actores que participaron en su formulación e implementación. En su dimensión funcional (o sea, la forma de funcionamiento del sistema en las instituciones prestadoras de servicios), se hace una evaluación de algunos aspectos de la atención farmacéutica y del uso racional de medicamentos por los pacientes, intentando también una aproximación al funcionamiento del sistema de abastecimiento de medicamentos en la base operativa del sistema de salud. Estas dos dimensiones están articuladas en el modelo estructural del nuevo Sistema de Salud establecido a partir de la reforma, con sus diferentes regímenes de afiliación (contributivo, subsidiado y vinculado), configurando una estrecha interrelación de condicionantes que inciden directamente sobre la equidad en el acceso a los medicamentos esenciales.

En el nivel macro, se investigó, mediante análisis documental, si el texto constitucional de Colombia insertaba implícita o explícitamente enunciados tendientes al logro de la equidad en la accesibilidad y el uso racional de medicamentos y cómo se reflejaban estos contenidos en las disposiciones de la Ley del SGSSS y de la PFN. En el mismo sentido, fueron analizados los textos reglamentarios y documentos gubernamentales emitidos a partir de la dación de la Ley. Se estudió también la percepción de 48 actores que desempeñaron funciones en el diseño, formulación e implementación de la PFN, en los distintos niveles y sectores: legislativo, ejecutivo (MS), organismos internacionales, directivos de EPSs, Instituciones Prestadoras de Salud (IPSs), Empresas Sociales del Estado (ESEs), Asociaciones de médicos y usuarios. Se aplicó la técnica de entrevista estructurada, indagando el papel que esos actores desempeñaron en el proceso, sus conceptos sobre acce- 
sibilidad y uso racional de medicamentos y sus recomendaciones para alcanzar la equidad en el sistema.

En el nivel micro, se evaluó la aplicación de la política en la dimensión relacionada al logro de la equidad en el acceso y en el uso racional de los medicamentos en dos ciudades. Fueron escogidas las ciudades de Medellín y Manizales, consideradas "ciudad grande" y "ciudad intermedia" respectivamente, en razón del número de habitantes y del nivel de desarrollo; y por posibilitar la comparación por contraste, dadas las diferencias de localización geográfica y régimen de afiliación en cada una de ellas. Se seleccionó la diabetes mellitus como enfermedad trazadora, por ser una patología de alta prevalencia en la población adulta y porque su diagnóstico y tratamiento farmacológico están estandarizados e incluidos en el POS.

A nivel institucional, se estudió el funcionamiento de algunos componentes del suministro de medicamentos: selección, programación, adquisición, distribución y dispensación de medicamentos. Las instituciones que formaron parte del estudio correspondieron a IPSs (47\%), ESEs (31,8\%), EPSs (9,1\%) y distribuidoras de medicamentos (4,5\%). Se seleccionaron aquellas que presentaban el mayor volumen de prestación de servicios y atendían la mayor demanda en los diferentes niveles de complejidad, totalizando 42 instituciones, que correspondían al 20\% del total en ambas ciudades: 7 en Manizales y 35 en Medellín.

En la encuesta a los médicos, fueron encuestados los prescriptores de pacientes diabéticos que laboraban en las instituciones seleccionadas, el $100 \%$ de médicos especialistas en Manizales (16 médicos) y el $80 \%$ de la ciudad de Medellín (70 médicos). Fueron explorados aspectos relacionados al conocimiento y uso de los medicamentos considerados en el POS para el tratamiento de diabetes mellitus, y a su conducta prescriptiva con los pacientes diabéticos, principalmente el uso de protocolos, información al paciente y reporte de reacciones adversas.

Con relación a los pacientes, fueron entrevistados 417 pacientes diabéticos (350 en Medellín y 67 en Manizales), seleccionados mediante muestreo sistemático simple, basados en una prevalencia de diabetes mellitus del 5\% en la población general. El tamaño muestral fue calculado con un 95\% de confianza y un error estándar de 5\%, y los aspectos considerados incluyeron:

1) Condiciones socio-económicas: género, nivel de escolaridad y de ingreso (menor a 1 Salario Mínimo Legal - SM L; entre 1-4 SMLs; entre 5-8 SM Ls; y mayor a 8 SMLs);
2) Cobertura del SGSSS y acceso: porcentaje y tipo de afiliación al sistema; procedencia;

3) Atención farmacéutica y prescripción: medicamentos prescritos (hipoglicemiantes orales e insulinas) y forma de prescripción; medicamentos del listado del POS disponibles en las instituciones (EPSs, ESEs, IPSs, Administradoras del Régimen Subsidiado - ARSs), medicamentos que deben ser adquiridos por el usuario y lugar donde se adquieren; prescripciones dispensadas por cuenta del POS; conocimiento del paciente sobre la diabetes y los medicamentos utilizados para su tratamiento; frecuencia y tipo de consulta de urgencias durante el último mes; prescriptor del medicamento; explicación recibida del médico sobre combinaciones, reacciones adversas de medicamentos; conocimiento sobre donde dirigirse en caso de error en el despacho o reacciones adversas; pacientes tratados conforme a protocolos definidos y número de medicamentos prescritos con nombre genérico.

En este trabajo, se adopta la definición de atención farmacéutica como el conjunto de actividades, de los comportamientos, los compromisos, las inquietudes, los valores éticos, las funciones, los conocimientos, las responsabilidades y las destrezas del farmacéutico en la prestación de la farmacoterapia, con el objeto de lograr resultados terapéuticos definidos en la salud y la calidad de vida del paciente (OMS, 1993). El acceso fue definido como la obtención de asistencia médica en caso de necesidad, y en relación a los medicamentos, como disponibilidad y posibilidad de obtención de los medicamentos prescritos, sea por distribución gratuita o mediante algún pago de bolsillo. Y la concepción de equidad adoptada fue la de ausencia de diferencias evitables, innecesarias y socialmente injustas (Daniels, 1998; Whitehead, 1992).

\section{Presentación de resultados y discusión}

La implementación de la PFN en el nuevo SGSSS y la equidad

La Constitución Colombiana de 1991 consagró el derecho a la seguridad social y a la salud, garantizando a todas las personas el acceso a los servicios de promoción, protección y recuperación de la salud (Asamblea Nacional Constituyente, 1991). Con relación a esto, el texto constitucional es taxativo, consagrándolo como servicio público de carácter obligatorio y con sujeción a los principios de eficiencia, universalidad y solidaridad, y asigna al Estado la 
función de facilitar las condiciones que garanticen el acceso de todos los habitantes a dicho servicio. Su sujeción al principio de universalidad es una referencia explícita a la equidad en su concepción igualitaria. El derecho a la salud en el texto se posiciona primero como un predicado del derecho a la vida y en segundo término con un carácter asistencial y prestacional. En su consagración, se hace una mención expresa a la equidad en el acceso.

Sin embargo, este acceso se da en la práctica por la afiliación a los diferentes regímenes, lo que depende en definitiva de las capacidades del sistema para afiliar a la población. El nuevo SGSSS en Colombia es de tipo mixto, y se caracteriza por la existencia de entidades públicas y privadas, que actúan de forma independiente, financiadas con recursos públicos. Entre los principales problemas, se identifican la duplicidad en la prestación de los servicios, la falta de coordinación por niveles de atención, el énfasis en la libertad del usuario para seleccionar la entidad prestadora de servicios, Ios costos elevados y la ausencia de racionalización de la oferta (Pérez, 1997).

En el texto constitucional, la palabra equidad es mencionada en tres oportunidades: la primera, refiriéndose a la imposibilidad de establecer controversias cuando haya sido invocada por el legislador; la segunda, invocando el deber ciudadano de contribuir al financiamiento de los gastos e inversiones del Estado bajo Ios conceptos de justicia y equidad; y la tercera, consagrando como función del Estado el velar por la distribución equitativa de oportunidades, adoptando medidas a favor de los grupos discriminados o marginados. Está implícito en la norma un ajuste de las oportunidades y recursos asignados a cada individuo en razón de diferencias, como sexo, ubicación geográfica, condiciones económicas, físicas o mentales, o individuos que se encuentren en circunstancias de debilidad manifiesta.

El principio de universalidad, consagrado en la ley, es un componente de equidad, toda vez que dispone la protección para todas las personas, sin discriminación en todas las etapas de la vida, y está íntimamente relacionado con la protección integral a la salud, prestada a través del POS que incluye atención preventiva, médico-quirúrgica y provisión de medicamentos esenciales. Para garantizar las condiciones de acceso de la población más pobre y vulnerable, el sistema crea el régimen subsidiado (Colombia, 1993).

Las disposiciones gubernamentales, como el Plan de Desarrollo 1998-2000 y específicamente aquellas tendientes a aumentar la co- bertura en el régimen subsidiado y beneficiar a ciertos grupos poblacionales - como: indigentes, niños abandonados y desplazados, considerando como criterios de inclusión el mayor índice de necesidades básicas insatisfechas (NBI) y los municipios con menores coberturas - favorecen el acceso de la población a la prestación de servicios de salud. Llama la atención la explícita referencia a procurar una disminución de las diferencias innecesarias e injustas mediante el acceso de la población a los servicios de salud, expresada en el propósito de ampliación de cobertura: con el fin de cumplir con los principios de equidad y obligatoriedad, mencionados en la Ley 100/ 1993.

Entretanto, se observa que hay contradicción abierta en la ley, al consagrar la universalidad y el acceso gradual al sistema - por disposición constitucional -, pués el objetivo de incorporar progresivamente al mayor número de personas y zonas geográficas como contribuyentes o como beneficiarios limita la aplicación del principio de universalidad, pues generalmente se pospone su aplicación hasta que se considere que están dadas las condiciones para hacerlo. Así mismo, la existencia de la población denominada "vinculados genera una indeterminación en cuanto al acceso a los servicios públicos de salud para este grupo, e implica también el no tener acceso a una provisión de medicamentos en caso de requerirlo. Tampoco es un predicado equitativo el disponer la ley sobre dos planes diferentes en salud: un POS para afiliados y beneficiarios del régimen contributivo y otro POS para la población beneficiaria del régimen subsidiado, el cual le da derecho a recibir un número menor de procedimientos e intervenciones. Así mismo, los copagos y las cuotas moderadoras consagrados como mecanismos de racionalizar el uso de los servicios o medicamentos, y sobre los cuales la ley es taxativa, pueden constituirse en obstáculos para el acceso y la provisión de servicios de salud en los diferentes niveles, sobretodo para la población pobre y vulnerable. Estos aspectos generan diferencias injustas y evitables.

Las cifras del MS mostraron, a junio de 1999, una cobertura del $40 \%$ de la población (aproximadamente 16 millones y medio); de ellos, el $30,5 \%$ pertenecía al régimen contributivo y 8,35\%, al régimen subsidiado (Salud Colombia, 1999a), cifras que revelan un aumento en la cobertura, pero se encuentran distantes de la meta de $100 \%$ propuesta en la ley. Así mismo, se habla de un retroceso en la cobertura del régimen contributivo (Salud Colombia, 1999b) el cual ha regresado a $30 \%$, posiblemente por efecto de la crisis económica, pues, a pesar de 
haber aumentado el porcentaje de aportes por nómina, el desempleo subió del 7 al 21\%, neutralizando así el valor de los recaudos; por otra parte, existen muchos casos de evasión fiscal, toda vez que los cotizantes declaran tener una renta menor para poder pagar menos. Es decir, la reforma, producto de las Leyes 60 y 100, no encontró un buen escenario para desarrollarse en un corto plazo, a parte que la capacidad de implementración y regulación de los organismos estatales es muy restringida. Además, a pesar del aumento logrado en la cobertura farmacéutica, se requiere un buen sistema de información para determinar si realmente las personas acuden a solicitar los servicios de salud y si reciben los tratamientos farmacológicos pertinentes.

En los decretos, resoluciones y acuerdos emitidos al respecto, se observa la intención del legislador de crear instrumentos que favorezcan la equidad en el acceso y el uso racional de medicamentos. La OMS (1998) destaca el hecho de que la accesibilidad y el uso racional de los medicamentos son parámetros que permiten medir la calidad de los servicios y se constituyen en parámetros de justicia y equidad. Se creó un Comité Técnico de Medicamentos (Consejo Nacional de Seguridad Social, 1997), como organismo asesor del CNSSS, con el objeto de estudiar y recomendar los criterios que orienten el proceso de actualización del listado, y se estableció una clasificación de los medicamentos, para programas especiales, manejo de patologías crónicas, de uso ambulatorio, hospitalario y medicamentos esenciales alternativos.

Se crearon también los Comités TécnicoCientíficos para actuar como mecanismo regulador de la demanda de los usuarios en las entidades administradoras y prestadoras de servicios de salud, y poder hacer ajustes en la prestación del servicio. Se destaca positivamentela disposición legal que autoriza la prescripción de medicamentos no incluidos en el listado, en aquellos casos en que, a criterio médico, se justifique el uso, con el fin de garantizar el derecho a la vida y a la salud de las personas (Consejo Nacional de Seguridad en Salud, 1998).

Entre las disposiciones que benefician el uso racional, están la creación del Programa de Medicamentos Esenciales y el uso de una lista de medicamentos para los diferentes niveles de atención, acompañados de la definición y de la adopción de protocolos de diagnóstico y tratamiento para las patologías contempladas en el POS. Estas acciones son fundamentales, considerando que fue comprobada la necesidad de limitar el número de medicamentos según las necesidades específicas del país o de la región, entre otras razones, para evitar un costo innecesariamente alto (OPS, 1998). También se fijaron disposiciones para la prescripción de medicamentos, que debe ser realizada por personal autorizado, por escrito, con previa evaluación del paciente e informando a éste las condiciones de prescripción, almacenamiento y medición de dosis.

Existen varios documentos sobre lineamientos de política farmacéutica expedidos por el Ministerio de Salud, sin que haya una claridad sobre cuál es el vigente, y el análisis realizado sobre aquellos considerados en este estudio permitió observar los siguientes avances:

1) Se expresa claramente que los lineamientos de la PFN deben concebirse como parte de una estrategia global de atención de la salud que privilegie el enfoque de promoción y prevención, y en la cual el uso racional de los medicamentos debe ser el criterio orientador de la atención farmacéutica. Se establece una concepción de atención farmacéutica integral, menos centrada en el medicamento como producto y más enfocada a la prestación de un servicio con calidad, eficiencia y efectividad.

2) Se promueven, en las diferentes instancias del sector farmacéutico - productores, prescriptores, distribuidores y consumidores-, la difusión del concepto de medicamento esencial y la utilización de la Denominación Común Internacional ( $\mathrm{DCl}$ ) o nombre genérico, con el fin de garantizar una oferta adecuada, reducir la prescripción inapropiada y mejorar el uso racional de los medicamentos basado en el perfil de morbi-mortalidad del país.

3) Se propicia el direccionamiento del mercado en función de aquellos medicamentos incluídos en el POS, y se propone estimular la producción nacional de medicamentos esenciales.

4) Se fijan estrategias para incentivar el uso racional, destacándose, entre ellas, la promoción de tratamientos no farmacológicos y de estilos de vida saludables, la creación de Comités de Farmacia y Terapéutica en las entidades administradoras y prestadoras de servicios de salud y la consolidación de los ya existentes.

5) EI MS se compromete a buscar alternativas diferentes a los controles administrativos de precios, favoreciendo mecanismos de mercado que, a través de una sana competencia, determinen franjas razonables de precios.

La percepción de los actores sobre la Política Farmacéutica Nacional

La mayoría de los actores entrevistados perciben que el diseño y formulación de la política 
favorece la accesibilidad, el uso racional de medicamentos y la equidad. También son unánimes en considerar la legislación adecuada y la política macro muy bien concebida, entretanto, señalan la falta de una mayor difusión, motivación, capacitación y control que garantice su correcta implementación.

Los actores hacen notar que la accesibilidad de la población a los servicios de salud y a los medicamentos esenciales está ligada a su afiliación al sistema y, en el caso de la población vinculada, no hay garantía de que puedan recibir estos servicios en sus diferentes niveles. Señalan que las barreras de tipo geográfico y económico, así como la ausencia de mecanismos de control, obstaculizan la implementación de la PFN. Se menciona también como un factor de influencia negativa la política de libertad de precios en el marco de libre mercado.

Discriminan además algunos factores relacionados al médico, al paciente y a los dispensadores que dificultan la racionalidad en el uso de medicamentos. En lo referente al médico, destacan la falta de conciencia de costos de los medicamentos; Ia influencia perjudicial de la publicidad y de la promoción farmacéutica en la práctica prescriptiva, caracterizada por un elevado uso de nombres de marca; deficiencias en la formación y capacitación durante el pregrado universitario; y el predominio del enfoque curativo de la atención en salud, en contraposición al de prevención y promoción. Según percepción de los actores entrevistados, tal como está diseñada la política farmacéutica, el listado básico impondría disciplina en la formulación de genéricos, pero el criterio individual del médico no se puede coartar y esto ha generado demandas a través de la tutela. Así mismo, observan que hay mayor sensibilidad del médico hacia el genérico en casos como la diabetes mellitus, no obstante, el uso del medicamento esencial se ha creado por obligatoriedad de la Ley 100, y no por una conciencia gremial sobre la importancia del uso racional. El hecho de existir un listado básico de medicamentos puede facilitar la entrega de algunos de ellos, pero a su vez es un obstáculo por la falta de cultura hacia el medicamento genérico por parte del médico, del paciente y de todo el personal de salud. Estos actores consideran que el listado debe dinamizarse a través de la actualización permanente, para evitar que se reduzca el acceso a medicamentos para patologías de mediana y alta complejidad.

Según Hodgkin \& Lanza (1999), en los países del Tercer Mundo hay pocas fuentes de producción y diseminación de información independiente, y el Estado y los gobiernos no alien- tan su existencia, todo lo contrario, socavan su existencia para evitar críticas. Según opinión de los actores, los médicos no tienen información adecuada respecto de los medicamentos esenciales, y destacan entre las razones la falta de educación continuada por parte de las universidades; la ausencia de actualización periódica del personal de salud en el manejo de los medicamentos y en la importancia de su racionalidad; y la influencia ejercida por los laboratorios farmacéuticos y la tecnología de punta. La educación médica continuada es responsabilidad de las universidades, las cuales han abandonado este campo, dejándolo en manos de los laboratorios farmacéuticos, debido, en parte, a la falta de coordinación que existe entre los organismos que regulan la política de medicamentos y las instituciones educativas. Con respecto a los pacientes, los actores consideran que hay una tendencia a exagerar en el uso de los medicamentos, no hay control en materia de automedicación y faltan políticas sobre educación a la comunidad con relación a los medicamentos en general.

Puntualizan la falta de información sobre el uso apropiado de los medicamentos, la concepción tergiversada del medicamento visto como un objeto de consumo cualquiera, y la práctica de automedicación. Finalmente, respecto a la entrega del medicamento por los dispensadores, mencionan: el incumplimiento de las normas sobre venta de medicamentos (con receta, venta libre, bajo control); la falta de capacitación del personal responsable; el predominio del interés lucrativo sobre el comportamiento ético en la función de dispensación, así como la falta de claridad sobre los parámetros para la substitución de los medicamentos prescritos.

Las instituciones analizadas y el cumplimiento de las directrices de la Política Farmacéutica Nacional

La Ley 100/93 dispuso que toda entidad distribuidora de medicamentos debía contar con un Comité Técnico Científico (MS, 1997b), el cual se encargaría de elaborar el listado básico de medicamentos para la institución y autorizar el suministro de medicamentos no contemplados en el POS. Sólo 38,6\% de las instituciones contaban con un Comité de Farmacia - principalmente en Manizales $y$, en menor proporción, en Medellín (Tabla 1) - y, de ese porcentaje, $18,2 \%$ daban instrucciones sobre los medicamentos que debían comprarse. Puede observarse que a nivel institucional no hay cumplimiento de la ley, posiblemente por falta de vigilancia por parte del Estado o, como opinan 
algunos formuladores de la PFN, porque falta operacionalizar la ley. Se observó, en la mayoría de instituciones (93,25\%), la presencia del listado del POS. Todas afirmaron realizar una programación de necesidades de medicamentos, general mente una vez por mes, habiendo recibido instrucciones sobre el tipo de medicamento que podían comprar (71,4\% en Manizales y $60 \%$ en Medellín). Los criterios utilizados para la adquisición son el uso de máximos y mínimos (45\%), el agotamiento (22,7\%), o ambos (11,4\%); el $20,9 \%$ de las instituciones no respondió la pregunta, porque no tenía criterios definidos. Hay una diferencia entre estos datos y los encontrados por Moreno et al. (1996), quienes reportaron $47 \%$ de utilización del método del consumo histórico para la programación de necesidades. Si esto ya era preocupante, por los problemas de confiabilidad de esa modalidad, la situación se agrava con los recientes hallazgos.

Una proporción importante de las instituciones expresó dificultades para la adquisición de medicamentos (77,3\%), relacionadas a la falta de presupuesto, a mecanismos de compras condicionadas o a la falta de oferta en el mercado, reconociendo también como un problema del sistema administrativo el incumplimiento en el pago a los laboratorios farmacéuticos. En el momento del estudio, se encontraron disponibles, en promedio, 7 de los 9 medicamentos esenciales seleccionados, coincidiendo con los hallazgos de González et al. (1997). Es importante analizar los procesos de adquisición y distribución de los medicamentos, porque es posible que en la formulación de la política se hayan tenido en cuenta las bases costo-efectivas para posibilitar el acceso; sin embargo, ante la ausencia de recursos económicos, un buen sistema de información y un adecuado sistema de suministro, no se puede aspirar a la equidad en el acceso a los medicamentos esenciales.

Se observó, en la mayoría de las instituciones, la prescripción de medicamentos incluídos en el POS (79\%), con diferencias porcentuales entre Manizales y Medellín (Tabla 1). El número de medicamentos por prescripción fue, en promedio, de dos, y $77 \%$ de los medicamentos fueron prescritos con su denominación genérica.

Sobre la dispensación de hipoglicemiantes orales e insulinas en las instituciones, 59,1\% correspondieron a aquellos incluídos en el POS, $31,8 \%$ fueron medicamentos del listado junto con otros no esenciales y $9,1 \%$ no pertenecían al POS. De los medicamentos despachados en las droguerías encuestadas, 41,5\% correspon- dían al listado del POS, 34,1\% combinaban medicamentos incluidos y no incluidos en el POS, $2,3 \%$ no eran del POS y $22,1 \%$ no contaban con los datos en el momento de la encuesta.

\section{La encuesta a los médicos}

Todos los médicos afirmaron conocer el Listado Básico del POS, la gran mayoría lo utilizaban, prescribiendo con nombre genérico $(61,1 \%)$ o en forma mixta (34,4\%) - nombre comercial y genérico -, y sólo 4,4\% lo hacen con nombre comercial. Al comparar los datos obtenidos en las dos ciudades, no se encontraron grandes diferencias (Tabla 2). Una proporción importante de los médicos (57,8\%) manifestó tener restricciones para prescribir medicamentos, sin embargo, 76,9\% no indicaron cuáles eran; los que respondieron señalaron entre ellas que sólo podían prescribir en genérico determinada dosis y por un tiempo determinado, o que sólo podían prescribir los medicamentos del listado, a pesar de utilizar protocolos que no los incluían.

En $88,9 \%$ de los casos, el médico considera que puede prescribir medicamentos fuera del listado del POS, en caso de necesidad, según

Tabla 1

Aspectos evaluados en las instituciones encuestadas, relacionados al uso racional de medicamentos, según localidad.

\begin{tabular}{lrrrr}
\hline $\begin{array}{l}\text { Aspectos evaluados } \\
\text { en las instituciones }\end{array}$ & \multicolumn{2}{c}{$\begin{array}{c}\text { Medellín } \\
\text { Sí (\%) }\end{array}$} & No (\%) & \multicolumn{2}{c}{ Manizales } \\
& & Sí (\%) & No (\%) \\
\hline Existencia de Comité de Farmacia & 28,6 & 71,4 & 85,7 & 14,3 \\
Presencia del listado del POS & 97,1 & 2,9 & 85,7 & 14,3 \\
Prescripción de medicamentos del POS & 81,7 & 18,3 & 62,9 & 37,1 \\
Prescripción en genérico & 79,6 & 20,4 & 62,5 & 37,5 \\
\hline
\end{tabular}

POS = Plan O bligatorio de Salud.

Tabla 2

Distribución del tipo de prescripción de medicamentos realizada por los médicos, según localidad de los pacientes diabéticos.

\begin{tabular}{lcc}
\hline Tipo de prescripción & Medellín (\%) & Manizales (\%) \\
\hline Genérico & 60,0 & 68,7 \\
Comercial & 4,3 & 6,3 \\
Mixto & 35,7 & 25,0 \\
Total & 100,0 & 100,0 \\
\hline
\end{tabular}


criterio médico y porque la ley lo permite previa solicitud y autorización de la institución. Entretanto, los que referieron que no podían prescribir fuera del listado (11,1\%) señalaron que era por problemas económicos de los pacientes o porque la entidad negaba los medicamentos prescritos.

Sobre el uso de protocolos de manejo, 73,3\% de los encuestados afirmaron que sí, los usaban (de Asociaciones o Sociedades Científicas, $33,3 \%$; de IPSs o EPSs donde laboran, $12,2 \%$; varios tipos de protocolos, $27,8 \%$ ), mientras, $26,7 \%$ no los usaban porque no conocían los protocolos, no existían protocolos en la institución, o los estaban desarrollando en el momento. Los porcentajes variaron según las ciudades, en Medellín 70\% de los médicos usaban protocolos y en Manizales, el 100\%.

La existencia y la utilización de protocolos de manejo por buena parte de los médicos favorecen el uso racional de los medicamentos porque permiten disminuir el número de medicamentos y priorizar las compras. Es importante destacar que, en Colombia, hay protocolos basados en la evidencia, elaborados por la Asociación Colombiana de Facultades de Medicina y por el Instituto de los Seguros Sociales (Molina et al., 1997). Sin embargo, 82,2\% de los médicos declararon recibir información sobre medicamentos (indicaciones, efectos adversos, cambios en las indicaciones, medicamentos nuevos); ante la pregunta de cómo se informaban, 32,2\% no contestaron, 38,9\% dijeron recibir información de los laboratorios farmacéuticos, $17,8 \%$, a través de la Internet, revistas o libros, $6,7 \%$, en eventos académicos, y un porcentaje menor a través de los colegas u otros medios. Esto podría indicar que no hay una adecuada difusión de los protocolos establecidos, o que dicha difusión llega con menor frecuencia a ciudades más alejadas. Con respecto a diferencias entre las ciudades Ilama la atención cómo en Medellín el porcentaje de médicos que reciben información de los laboratorios farmacéuticos $(45,7 \%)$ es muy alto, mientras, en Manizales, la mayoría de los médicos encuestados dicen recurrir a la Internet, a bases como el MEDLINE, a revistas o libros (50\%).

Una proporción importante de médicos $(84,4 \%)$ manifestó que ellos explicaban a sus pacientes diabéticos sobre los medicamentos (uso, contraindicaciones, reacciones adversas, interacciones y posología). Sólo $28,9 \%$ de los médicos señalaron que sabían donde informar las reacciones adversas de los medicamentos. De ellos, 26,9\% no respondieron donde hacerlo, el resto afirmó que informarían a la institución donde laboran (26,9\%), al Laboratorio de
Toxicología de la Universidad (23,1\%), al INVIMA (7,7\%), al Laboratorio Farmacéutico productor $(7,7 \%)$ o a las Direcciones Seccionales de Salud (3,8\%). El bajo porcentaje de médicos que sabe dónde reportar las reacciones adversas a medicamentos se puede explicar porque el programa de farmacovigilancia, que debe ser coordinado por el INVIMA, aún está en fase de desarrollo y sólo se han realizado pruebas piloto en Santafé de Bogotá (Denis et al., 1998).

\section{La encuesta a los pacientes}

El 77,2\% de los pacientes diabéticos encuestados estaba afiliado al SGSSS, de ellos, el $48,6 \%$ pertenecía al régimen contributivo, $28,6 \%$ al régimen subsidiado, el $19,8 \%$ era vinculado y el $3 \%$ era particular o pertenecía a regímenes especiales. Esta cobertura es mayor a la encontrada por Cardona et al. (1999) en la población general, en diferentes regiones del país, señalando $30,8 \%$ para el régimen contributivo y $18,3 \%$ para el subsidiado.

Es interesante observar que el porcentaje de pacientes afiliados al régimen contributivo sea mayor en una ciudad intermedia como Manizales (64,2\%), con respecto a Medellín (43,7\%). La distribución para el régimen subsidiado es de $20,9 \%$ de pacientes diabéticos en $M$ anizales y 31,4\% en Medellín. Se observa aumento de cobertura según régimen en Medellín y Manizales, sin embargo, si consideramos que el contributivo aporta el $1 \%$ para el subsidio en salud, se hace evidente la desigualdad entre ambas ciudades, pues, en Medellín el Estado tiene que asumir los costos para el 53,7\% de la población (subsidiada más vinculada) mientras en Manizales lo hace para el $29,8 \%$.

No se presentaron diferencias importantes entre los grupos de edad en función del régimen de afiliación, pero se observó que la mayoría de los pacientes se encontraba en el grupo de 60 años o más, lo que era de esperarse puesto que la diabetes mellitus se presenta con mayor frecuencia en pacientes de edad avanzada, o es posible que los pacientes diabéticos al pertenecer a las asociaciones respectivas o al estar incluidos en programas de promoción y prevención consideren necesaria la afiliación al SGSSS. Es importante anotar que Cardona et al. (1999) encontraron disminución en la afiliación de personas mayores de 60 años en la población general.

El nivel educativo de la mayor parte de los pacientes era educación básica primaria (49,5\%), observándose proporciones mayores en los regímenes subsidiado $(62,1 \%)$ y vinculado (50\%) que en el régimen contributivo $(43,1 \%)$. 
Este dato es importante, considerando que el grado de educación influye en la actitud hacia la promoción y la prevención de la enfermedad, así como en el cumplimiento de las prescripciones y en el uso de medicinas alternativas.

Al analizar el nivel de ingresos de los pacientes encuestados, se encontró que, en la ciudad de Medellín, la mayoría $(47,7 \%)$ recibían menos de un SML y a su vez, pertenecían al régimen contributivo. En Manizales, 56,7\% recibían entre uno y cuatro SML y $64,2 \%$ pertenecían al régimen contributivo. Llama la atención que pacientes con bajo nivel educativo y menos de un SML de ingreso pertenezcan al régimen contributivo, el cual está diseñado para personas que devenguen un SML o más. Estos datos coinciden con los del estudio de Cardona et al. (1999) que encontraron una baja cobertura en pacientes de bajos recursos, debido posiblemente a que los afiliados pertenecían al régimen contributivo por ser asalariados.

Con relación a la dispensación de medicamentos, 56,5\% de los pacientes diabéticos no recibieron los medicamentos correspondientes, hipoglicemiantes orales e insulinas incluidos en el POS, 34\% de estos pacientes pertenecían al régimen contributivo y $22,5 \%$, al régimen subsidiado. Del $43,5 \%$ de los pacientes a quienes les despacharon los medicamentos sin costo, la gran mayoría pertenecían al régimen contributivo, una sexta parte, al régimen subsidiado y una muy pequeña proporción era vinculada (Tabla 3). La no dispensación de medicamentos a los pacientes puede deberse a fallas en la selección de los medicamentos, en la programación de necesidades, en la compra, en la gestión del suministro; a las condiciones establecidas por los laboratorios farmacéuticos para el abastecimiento; o responder a problemas económicos de las instituciones, ya sea por la intermediación generada en el nuevo sistema, el diseño del flujo de recursos o la política de reducción de costos. Se deben ejercer mecanismos de control que permitan asegurar la existencia de un stock de inventarios, estándares mínimos para reposición, la obligatoriedad en la entrega y la creación de canales de distribución más amplios. Los datos obtenidos con relación a esto muestran que los pacientes diabéticos, a pesar de estar afiliados al sistema de salud, sólo tienen acceso teórico a los medicamentos esenciales del POS; en la práctica, el usuario no tiene agilidad en la dispensación de los medicamentos.

En teoría, el 77,2\% de los pacientes tiene derecho a recibir el tratamiento farmacológico sin costo, al estar cubiertos por el sistema, pero en la entrega del medicamento no sólo influye el derecho al servicio y a la atención en salud, sino la disponibilidad oportuna del mismo. Precisamente, el estudio muestra como la principal causa para el no despacho de medicamentos sin costo la negativa de la entidad aseguradora para suministrarlo o la exigencia de un pago parcial. La mayoría de los pacientes diabéticos (56,2\%) debieron adquirir por su cuenta los medicamentos incluidos en el POS. Esa situación indica una violación a la Ley 100/ 93 por parte de las entidades responsables, al negar a los pacientes el suministro de medicamentos con cargo a la unidad de pago por capitación, bajo criterios de calidad y oportunidad (MS, 1997a). Y representa un problema grave, si se considera que el mayor porcentaje de pacientes diabéticos que no reciben medicamentos pertenecen al régimen contributivo, grupo que, en el estudio, también se caracterizó por el bajo nivel económico de las personas, cuyos ingresos familiares, en su mayoría, sólo alcanzan un SML, estando sujetos además al pago de cuotas moderadoras y copagos. Según percepción de los actores entrevistados, Ias cuotas moderadoras y los copagos pueden crear una barrera para las clases menos favorecidas, las cuales se ven seriamente afectadas, una vez que la accesibilidad está ligada a la afiliación al SGSSS en Colombia.

Los pacientes adquirieron los medicamentos ( Tabla 4) con mayor frecuencia en las asociaciones de diabéticos y farmacias $(54,1 \%)$ y, en menor porcentaje, en las EPSs, IPSs, ESEs y ARSs (45,9\%). En ambas ciudades, Ios pacientes que adquirieron los medicamentos en las asociaciones de diabéticos pertenecían, en su mayoría, al régimen subsidiado o eran vinculados. Los que adquirieron los medicamentos en las farmacias eran principalmente del régimen contributivo.

Ante la falta de disponibilidad de medicamentos en el SGSSS, el sector privado juega un importante papel en la adquisición de los me-

Tabla 3

Dispensación de medicamentos, sin costo, a los pacientes diabéticos, según régimen de afiliación.

\begin{tabular}{lccc}
\hline $\begin{array}{l}\text { Régimen } \\
\text { de afiliación }\end{array}$ & $\begin{array}{c}\text { Medicamentos no } \\
\text { dispensados (\%) }\end{array}$ & $\begin{array}{c}\text { Medicamentos } \\
\text { dispensados (\%) }\end{array}$ & Total (\%) \\
\hline Contributivo & 34,0 & 34,0 & 68,0 \\
Subsidiado & 22,5 & 7,5 & 30,0 \\
Vinculado & 0,0 & 2,0 & 2,0 \\
Total & 56,5 & 43,5 & 100,0 \\
\hline
\end{tabular}


Tabla 4

Sitio de adquisición de los medicamentos no dispensados por parte de los pacientes diabéticos.

\begin{tabular}{lcr}
\hline Sitio de adquisición & Frecuencia & $\%$ \\
\hline Asociación de diabéticos & 108 & 24,88 \\
Droguerías particulares & 127 & 29,26 \\
Empresas Sociales del Estado & 81 & 18,66 \\
Instituciones Prestadoras de Salud & 53 & 12,21 \\
Empresas Promotoras de Salud & 49 & 11,29 \\
Administradoras del Régimen Subsidiado & 16 & 3,69 \\
Total & 434 & 100,00 \\
\hline
\end{tabular}

dicamentos por parte de los pacientes, sin embargo, no garantiza una atención farmacéutica completa que incluya educación e información al paciente, tal como está contemplado en la ley; además de afectar el presupuesto de los pacientes, a quienes les genera sobrecostos. Ante la falta de disponibilidad de medicamentos en el SGSSS, el sector privado juega un importante papel en la adquisición de los medicamentos por parte de los pacientes, sin embargo, no garantiza una atención farmacéutica completa que incluya educación e información al paciente, tal como está contemplado en la ley; además de afectar el presupuesto de los pacientes, a quienes les genera sobrecostos.

Se encontró también que $70 \%$ de los pacientes diabéticos tienen conocimiento sobre su enfermedad, la identifica como una enfermedad grave, de cuidado, relacionada con la herencia, que puede además afectar muchos órganos vitales. Además, la mayoría de ellos afirman tener conocimiento sobre su tratamiento, lo que coincide con los datos obtenidos de los médicos entrevistados, quienes declararon dar información a sus pacientes. Sin embargo, se debe recordar la baja escolaridad del paciente diabético encuestado, lo que puede llevar a una consulta de urgencia, porque el paciente no se apropia del conocimiento. En promedio, $20,3 \%$ de los pacientes requirieron consulta de urgencia durante el último mes.

En la Ley 100/93 se considera, la importancia de los programas permanentes para la atención preventiva de enfermedades y riesgos específicos, donde se brinde orientación a los afiliados sobre la forma de reconocer la oportunidad para solicitar la atención por consulta externa o urgencias, identificar signos o síntomas de alerta y la importancia del uso racional de los medicamentos formulados por profesionales responsables. En estos aspectos no se ob- servan diferencias entre los pacientes diabéticos respecto a la información que reciben según régimen y ubicación geográfica.

El 54,1\% de los pacientes diabéticos recibió información por parte del médico sobre los efectos al combinar los hipoglicemiantes orales e insulinas, la mayoría declaró saber dónde debe reportar reacciones adversas, entretanto, sólo el 48,1\% sabía dónde quejarse si le cambian el medicamento (entidad prestadora del servicio, farmacia o médico tratante). Medellín presentó mayor porcentaje de pacientes informados sobre interacciones con medicamentos, reporte de reacciones adversas, qué hacer en caso de cambio de medicamento y utilidad de los mismos.

Los medicamentos prescritos pertenecían al listado del POS en $91,5 \%$ de los casos. De los pacientes encuestados, 40,4\% manifestaron haber recibido prescripciones con nombre comercial - lo que se observó con mayor frecuencia en Manizales (62,7\%) que en Medellín (10,3\%) -, de ese porcentaje 34,6\% pertenecían al régimen contributivo y $47,6 \%$ al subsidiado; 18,8\% afirmaron que la prescripción había sido en genérico y $2,5 \%$ en forma mixta. Entretanto, $38,3 \%$ de ellos no respondieron a la pregunta, lo que podría indicar poco conocimiento sobre la diferencia entre los nombres genérico y comercial de los medicamentos formulados. Este aspecto es importante, porque incide en el costo de adquisición para el paciente, sobretodo considerando que la diabetes mellitus es una enfermedad crónica. Interesa resaltar también aún cuando la mayoría de pacientes encuestados están amparados en la Ley 100/ 93 por pertenecer al régimen contributivo; los medicamentos fueron prescritos con nombre comercial, siendo la promoción del uso de los medicamentos esenciales en su denominación genérica un tema de énfasis del MS para el período 1995- 1998.

Adicionalmente, el 52,8\% de los pacientes encuestados recurre a medicinas alternativas para el tratamiento de la diabetes, como el uso de plantas medicinales, acupuntura y orinoterapia. A pesar de que en la formulación de la Ley 100/93 se facilitó el acceso a los medicamentos, los comportamientos, las actitudes y las creencias de los pacientes tienen un gran peso en la utilización de los servicios de salud y de los medicamentos. En Colombia, está muy arraigado el uso de medicinas alternativas y la automedicación, y generalmente, cuando el paciente acude al médico, ya ha consultado a los vecinos, amigos o herbolarios, sin considerar el peligro de utilizar cualquier sustancia, sin información, desconociendo su real eficacia, 
Ias concentraciones requeridas, el tiempo de uso y sobretodo las potenciales reacciones adversas y efectos secundarios.

\section{Conclusiones y recomendaciones}

Esta investigación fue orientada a la evaluación de algunos resultados de las medidas aplicadas en la implementación de la Política Farmacéutica Nacional en Colombia, en el marco de la reforma en salud.

La estructura legal es parte del soporte de cualquier política y, específicamente en este caso, se han encontrado claros lineamientos de política farmacéutica en la legislación desarrollada alrededor de la reforma del sistema de salud o en documentos elaborados por el MS.

En ese sentido, es un avance que la garantía de acceso a medicamentos esenciales por la población sea considerada en la Ley Marco de Salud, porque la atención farmacéutica debería siempre estar contemplada dentro de la política nacional de salud como parte de las acciones de prevención, promoción y recuperación consideradas en la atención sanitaria. Sin embargo, la propia naturaleza del medicamento trasciende el aspecto sanitario, congregando otras esferas de la dinámica nacional, que lo convierten en un objeto diferenciado sobre el cual es necesario plantear el desarrollo de una política específica, de desarrollo científico y tecnológico.

El modelo adoptado en la reforma colombiana del sector salud presenta, en su estructura, condicionantes que generan diferencias socialmente injustas, al distinguir los grupos de población afiliados al sistema en función de su capacidad de pago (contribuyentes, subsidiados, vinculados). Aúnque especifique también mecanismos de solidaridad entre los regímenes, las dificultades de implementación y de regulación acaban por caracterizarse como determinantes estructurales de inequidad en el SGSSS, lo cual se refleja en las diferencias de cobertura en la prestación de servicios de salud $\mathrm{y}$, consecuentemente, en los bajos niveles de acceso a medicamentos esencial es por parte de la población subsidiada y vinculada.

La implantación aún incipiente de la PFN en el contexto del proceso de reforma ha promovido e impulsado algunos avances importantes en la atención farmacéutica, los cuales encuentran limitantes en la propia estructura del sistema que reproduce las desigualdades socio-económicas en las barreras de acceso a los servicios y bienes esenciales de salud. Por otro lado, su implementación aún no ha con- seguido un efecto transformador en las prácticas de trabajo y en el mejoramiento de la gestión en el suministro de medicamentos esenciales.

Final mente, el elevado uso de medicina alternativa por los pacientes Ilama la atención para el transfondo cultural en la relación de adecuación usuario-prestador, generalmente no considerada en los estudios clási cos de evaluación en salud. Más aún, tratándose del medicamento, es indispensable observar el valor simbólico atribuido a él en el imaginario de la población, para poder comprender y orientar su comportamiento en relación al uso adecuado y racional.

Con base en los resultados de la investigación, se recomienda:

- Implementar una política integral de medicamentos (o Ley del Medicamento) que fusione y fortal ezca todas las estrategias relacionadas con la producción, regulación, suministro y uso de los medicamentos.

- Trabajar en estrategias que permitan disminuir la diferencia en la accesi bilidad y en el uso de medicamentos entre las ciudades y entre los diferentes usuarios del régimen de seguridad social.

- Vigilar la creación y puesta en marcha de los Comités técnico-científicos en las diferentes instituciones pertenecientes al SGSSS.

- Incentivar el uso de información normalizada (protocolos) entre la población médica del SGSSS.

- Fomentar la educación continuada para los profesionales de la salud en lo referente a medicamentos esencial es y presentaciones genéricas.

- Sugerir a las universidades la inclusión, en el plan de estudios de pregrado, de postgrado y en educación continuada a los egresados, del componente de medicamentos esenciales con temas como buenas prácticas de prescripción, protocolos y guías de tratamiento.

- Fomentar la difusión sobre los derechos y deberes en materia de medicamentos en el SGSSS.

- Aplicar los mecanismos de vigilancia y control por parte de las entidades encargadas de esta función.

- Divulgar los resultados de la investigación a las instituciones, entidades de dirección y control de la política de medicamentos. 


\section{Agradecimientos}

Este proyecto fue desarrollado en la Universidad de Caldas, Facultad de Ciencias para la Salud, Departamento de Ciencias Básicas de la Salud, Manizales, Colombia, como parte del Programa Equity-oriented Health Policy Analysis in Latin America, implementado por la Red de Investigación en Sistemas y Servicios de Salud en el Cono Sur de América Latina, financiado por el Internacional Development Research Center, de Canadá. Agradecemos el soporte técnico brindado por Nadine Gassman, Carmen Phang Romero, asistente de investigación de la Red y Celia Almeida, Coordinadora del Programa y Secretaria Ejecutiva de la Red.

\section{Referencias}

ASAMBLEA NACIONAL CONSTITUYENTE, 1991. Constitución Política de Colombia 1991. Gaceta Constitucional. № 116. Bogotá: Asamblea Nacional Constituyente.

COLOMBIA, 1993. Ley № 100, del 23 de diciembre de 1993. Crea el Sistema de Seguridad Social Integral y se dictan otras disposiciones. Bogotá: Diario Oficial del Congreso dela República de Colombia. Año CXXIX, № 41148, p. 40.

COLOMBIA, 1994. Decreto № 1290, del 22 de junio de 1994. Precisan las funciones del Instituto Nacional de Vigilancia de Medicamentos y Alimentos INVIMA - y se establece su organización básica. Bogotá: Diario Oficial de la República de Colombia. Año CXXX, № 41406, 24, p. 17.

COLOMBIA, 1995. Decreto № 123, del 13 de enero de 1995. Aprueba el Acuerdo № 2, por el cual se establecen la estructura interna y las funciones de las dependencias del Instituto Nacional de Vigilancia de Medicamentos y Alimentos - INVIMA y se dictan otras disposiciones. Bogotá: Diario Oficial dela República de Colombia, Año CXXX, № 41676, p. 14.

COLOMBIA, 1996. Decreto № 2174, del 28 de noviembre de 1996. Sistema obligatorio de garantía de calidad del Sistema General de Seguridad Social en Salud. Bogotá: Diario Oficial dela República de Colombia, Año CXXXII, no 42931, 3 diciembre, p. 9.

CONSEJO NACIONAL DE SEGURIDAD SOCIAL, 1997. Acuerdo № 51, del 8 de enero de 1997. Crea el ComitéTécnico en Medicamentos y se Dictan Normas sobre su Funcionamiento. Bogotá: Ministerio de Salud.

CONSEJO NACIONAL DE SEGURIDAD EN SALUD, 1998. Acuerdo № 110, del 11 de septiembre de 1998. Modifica el Acuerdo № 83 del Consejo Nacional de Seguridad en Salud. Bogotá: Boletín № 012,5, p. 80.
CARDONA, A.; NIETO, F.; ARBELAEZ, M .; AGUDELO, H.; CHAVEZ, B.; MONTOYA, A. \& ESTRADA, A., 1999. Impacto de la reforma de la seguridad social sobre la organización institucional y la prestación de los servicios de salud en Colombia. Public Health Journal of the Net, Agosto/Septiembre. Febrero $2000<$ tttp:// www.saludcolombia.com/ actual/salud39/informe.htm>.

DANIELS, N., 1998. Parámetros de justicia y monitoreo de la equidad: Apoyo a un programa de la OMS. Salud y Gerencia, 16:7-12.

DENIS, R.; GUTIERREZ, J. \& RODRIGUEZ, M., 1998. Creación de un Programa Piloto de FarmacovigiIancia en el Hospital Universitario de San Ignacio. Acta Medica Colombiana, 23:15-21.

GONZALEZ, E.; ROSSI, F.; ARANGO, J. I. \& MARIN, N., 1997. Analysis of the National Drug Policy of Colombia. Final Report. Project: ComparativeAnalysis of the National Drug PolicyWHO - Karolinska - Harvard. Bogotá: Páramo Gómez.

HODGKIN, C. \& LANZA, O., 1999. Tercer mundo y el acceso a medicamentos esenciales. Fármacos, Septiembre 1999. 28 Febrero 2000 \ttp:// www. farmc.utexas.edu/ project/ fármacos/999com01. htm>.

INS (Instituto Nacional de Salud), 1980. El Gasto Privado en Salud. Bogotá: Ministerio de Salud.

MOLINA, C., 1993. El Gasto Público en Salud y Distribución de Subsidios en Colombia. Bogotá: Fedesarrollo. (mimeo.)

MORENO, R.; JARAMILLO, C.; ARBELAEZ, M.; CAÑAS, B.; CONTRERAS, C.; GOMEZ, M.; RESTREPO, M.; SANTA, T.; TORO, V. \& VALLEJO, A., 1996. Análisis dela Política Nacional de Medicamentos en CoIombia. 1995. OMS/OPS. Centro Colaborador en el área de Sistemas y Suministro de medicamentos e insumos hospitalarios esenciales. Medellín: Cooperativa de Hospitales de Antioquia.

MS (Ministerio de Salud), 1995. Lineamientos para la 
Formulación dela Política Farmacéutica Colombiana en el Sistema de Seguridad Social en Salud. Cartagena: MS.

MS (Ministerio de Salud), 1996. Lineamientos para la Formulación dela Política Farmacéutica Colombiana en el Sistema de Seguridad Social en Salud. Bogotá: MS.

MS (Ministerio de Salud), 1997a. Acuerdo № 83, del 23 de diciembre de 1997. Adopta el manual de medicamentos del Sistema General de Seguridad Social en Salud. Boletín Jurídico, 10:219-220.

MS (Ministerio de Salud), 1997b. Resolución № 05061, del 23 de diciembre de 1997. Reglamenta los Comités Técnico-Científicos dentro de las entidades promotoras de salud, administradoras del régimen subsidiado e instituciones prestadoras de servicios de Salud, y se dictan otras disposiciones. In: Medicamentos a los que Ud. tiene Derecho en el Sistema General de Seguridad Social en Salud, (Ministerio de Salud, org.), pp. 49-50, Bogotá: MS.

OMS (Organización Mundial de la Salud), 1993. El papel del farmacéutico en el sistema de atención de salud. In: Informe de la Reunión de la OMS en Tokio, Japón OMS, org.), p. 3, Geneva: OMS.

OMS (Organización Mundial de la Salud), 1998. Actividades de la OMS en Relación con las Políticas Farmacéuticas y los Medicamentos Esenciales. Geneva: OMS.

OPS (Organización Panamericana de la Salud)/OMS (Organización Mundial de la Salud), 1998. Estrategia Revisada dela OMS en Materia de Medicamentos. Washington, DC: OPS.
OROSTEGUI, M., 1990. Situación de salud. In: La Salud en Colombia. Estudio Sectorial de Salud (F. Yepes, org.), pp. 219-467, Bogotá: Departamento Nacional de Planeación, Ministerio de Salud.

PEREZ, P. H., 1997. Sistemas Nacionales de Salud. In: Fundamentos de Salud Pública (J. H. Blanco \& J. M. Maya, org.), pp. 36-42, Medellín: Corporación para Investigaciones Biológicas.

SALUD COLOMBIA, 1999a. Las EPS a junio de 1999. Public Health Journal of the Net, Octubre/ Noviembre 1999. February $2000<$ বttp:// www.salud colombia.com/actual/salud41/informe.htm>

SALUD COLOMBIA, 1999b. Reportaje. Public Health Journal of the Net, Octubre/ Noviembre. Febrero 2000 বttp:// www.saludcolombia.com/actual/ salud39/ report.htm>

TONO, T., 2000. La Evolución del Acceso a la Salud en Colombia. ¿Subsidios a la Oferta o a la Demanda? Vía Salud. Segundo trimestre 12. Bogotá: Centro de Gestión Hospitalaria.

WHITEHEAD, M., 1992. The concepts and principles of equity in health. International Journal of Health Services, 22:429-445.

YEPES, J. F. (org.), 1990. La Salud en Colombia. Estudio Sectorial de Salud, Tomo I y II. Bogotá: Departamento Nacional de Planeación, Ministerio de Salud.

Recibido el 24 de mayo de 2001

Versión final presentada el 18 de septiembre de 2001

Aprobado el 4 de diciembre de 2001 